\title{
Perfil dos pacientes e dos terceiros molares extraídos na Faculdade de Odontologia
}

\section{de Araçatuba - UNESP}

\author{
Profile of patients and third molars extracted at the Faculty of Dentistry of Araçatuba - UNESP \\ Perfil de pacientes y terceros molares extraídos en la Facultad de Odontología de Araçatuba -
}

Osvaldo Magro Filho

ORCID: https://orcid.org/0000-0002-9821-2479 Universidade Estadual Paulista, Brasil

E-mail: osvaldo.magro@unesp.br

Idelmo Rangel Garcia Júnior

ORCID: https://orcid.org/0000-0001-8892-781X Universidade Estadual Paulista, Brasil

E-mail: irgcirurgia@gmail.com

Alessandra Marcondes Aranega

ORCID: https://orcid.org/0000-0001-5856-7972

Universidade Estadual Paulista, Brasil

E-mail: alessandra@ foa.unesp.br

Francisley Ávila Souza

ORCID: https://orcid.org/0000-0002-1427-071X

Universidade Estadual Paulista, Brasil

E-mail: francisley.avila@unesp.br

Ana Paula Farnezi Bassi

ORCID: https://orcid.org/0000-0002-0031-4953

Universidade Estadual Paulista, Brasil

E-mail: apfarnezibassi@gmail.com

Leonardo Perez Faverani

ORCID: https://orcid.org/0000-0003-2249-3048 Universidade Estadual Paulista, Brasil

E-mail: leonardo.faverani@unesp.br

Daniela Ponzoni

ORCID: https://orcid.org/0000-0001-5928-0914

Universidade Estadual Paulista, Brasil

E-mail: daniela.ponzoni@unesp.br

\begin{abstract}
Resumo
Na área odontológica de cirurgia bucomaxilofacial um grande número de casos de dentes não erupcionados e impactados é comumente encontrado nos pacientes atendidos na Faculdade de Odontologia de Araçatuba - UNESP. O objetivo deste estudo foi determinar o perfil dos pacientes e dos terceiros molares indicados para exodontia, assim como seu tratamento cirúrgico. Foram obtidos para a pesquisa o perfil dos pacientes e dos dentes extraídos a partir de tratamentos realizados nas clínicas da faculdade, onde uma ficha foi aplicada para cada dente extraído. A partir das fichas foram obtidos os dados pessoais dos pacientes, a presença ou não de doença sistêmica, classificação de Pell \& Gregory e Winter para terceiros molares, se o dente está causando algum transtorno mecânico, nervoso, infeccioso ou tumoral. Consta também preenchimento na ficha para o período pós-operatório se existiu ou não algum tipo de
\end{abstract}


transtorno. Ao longo de três anos foram atendidos 134 pacientes, sendo $57 \%$ do sexo feminino e $43 \%$ do sexo masculino, tendo a maior procura por atendimento a faixa etária de 20 a 29 anos, totalizando 275 dentes extraídos. Em $54 \%$ dos casos foram extraídos molares inferiores, sendo a posição A e a classe II mais prevalentes segundo a classificação de Pell \& Gregory. Para todos os terceiros molares extraídos a posição mais frequente foi a vertical com $58 \%$ dos casos segundo a classificação Winter. Conclui-se que a maioria dos pacientes são jovens do sexo feminino prevalecendo os procedimentos de exodontia de terceiros molares inferiores. E as complicações pós-operatórias mais frequentes foram o edema, alveolite e parestesia do nervo alveolar inferior.

Palavras-chave: Cirurgia bucal; Terceiro molar; Dente não-erupcionado.

\begin{abstract}
In the dental area of maxillofacial surgery, a large number of cases of unerupted and impacted teeth is commonly found in patients seen at the Araçatuba Dental School - UNESP. The aim of this study was to determine the profile of patients and third molars indicated for extraction, as well as their surgical treatment. For the research, the profile of patients and teeth extracted from treatments carried out in the college's clinics were obtained, where a form was applied to each extracted tooth. The personal data of the patients were obtained from the files, the presence or not of systemic disease, Pell \& Gregory and Winter classification for third molars, if the tooth is causing any mechanical, nervous, infectious or tumoral disorder. There is also filling in the form for the postoperative period whether or not there was any type of disorder. Over three years, 134 patients were seen, $57 \%$ female and $43 \%$ male, with the greatest demand for care being in the age group of 20 to 29 years, totaling 275 extracted teeth. In $54 \%$ of the cases, mandibular molars were extracted, with position A and class II being more prevalent according to Pell \& Gregory's classification. For all extracted third molars, the most frequent position was the vertical with $58 \%$ of cases according to the Winter classification. It is concluded that most patients are young females, with lower third molar extraction procedures prevailing. Moreover, the most frequent postoperative complications were edema, alveolitis and inferior alveolar nerve paresthesia.
\end{abstract}

Keywords: Oral surgery; Third molar; Unerupted tooth.

\title{
Resumen
}

En el área dental de la cirugía maxilofacial, es común encontrar un gran número de casos de dientes impactados y no erupcionados en pacientes atendidos en la Facultad de Odontología de Araçatuba - UNESP. El objetivo de este estudio fue determinar el perfil de los pacientes y terceros molares indicados para la extracción, así como su tratamiento quirúrgico. Para la investigación se obtuvo el perfil de pacientes y dientes extraídos de tratamientos realizados en las clínicas de la facultad, donde se aplicó un formulario a cada diente extraído. Los datos personales de los pacientes se obtuvieron de los expedientes, presencia o no de enfermedad sistémica, clasificación de Pell \& Gregory y Winter para terceros molares, si el diente es causante de algún trastorno mecánico, nervioso, infeccioso o tumoral. También hay que cumplimentar el formulario del postoperatorio si existió o no algún tipo de trastorno. A lo largo de tres años se atendieron 134 pacientes, 57\% mujeres y $43 \%$ hombres, con mayor demanda de atención en el grupo de edad de 20 a 29 años, totalizando 275 dientes extraídos. En el 54\% de los casos se extrajeron molares mandibulares, siendo la posición A y la clase II las más prevalentes según la clasificación de Pell \& Gregory. Para todos los terceros molares extraídos, la posición más frecuente fue la vertical con un $58 \%$ de los casos según la clasificación de Winter. Se concluye que la mayoría de los pacientes son mujeres jóvenes, predominando los procedimientos de extracción del tercer molar inferior. Y las complicaciones postoperatorias más frecuentes fueron edema, alveolitis y parestesia del nervio alveolar inferior.

Palabras clave: Cirugía bucal; Tercer molar; Diente intacto.

\section{Introdução}

As cirurgias de dentes inclusos são atos cirúrgicos realizados rotineiramente em consultórios e na Faculdade de Odontologia de Araçatuba - UNESP. A realização destes procedimentos envolve conhecimentos específicos e treinamento adequado, pois um protocolo de extração inadequado pode consequentemente ocasionar complicações trans e pós-operatórias que não devem ser desprezadas (Khojastepour et al., 2019).

Um dente incluso é aquele em processo de erupção ou que se apresenta impactado. Por sua vez, um dente impactado é assim chamado quando não consegue irromper devido à presença de alguma barreira física. Os dentes tornam-se frequentemente impactados porque o comprimento total do arco ósseo alveolar é menor que o comprimento total da arcada dentária, fazendo com que os dentes com erupção mais tardia não encontrem espaço para sua acomodação adequada. Dessa forma, os dentes usualmente impactados são os terceiros molares inferiores e superiores, seguidos pelos caninos superiores e dentes supranumerários (Xavier et al., 2010). 
Após passado o seu período normal de erupção, permanece alojado no rebordo alveolar da maxila ou mandíbula recoberto por mucosa e ou tecido ósseo. Depreende-se desse conceito que, as inclusões dentárias, de forma geral, podem ser classificadas como: intra-óssea, semi-incluso ou submucoso. O dente intra-ósseo é que se apresenta totalmente circundado por osso. Quando esse elemento perfura a cortical óssea, ainda permanece incluso e com a mucosa suprajacente íntegra, denominamos de submucoso. E quando esse dente se comunica com a cavidade oral, porém, devido a sua inclusão, não atinge a irrupção completa, recebe a denominação de semi-incluso (Medeiros et al., 2003).

A remoção dos dentes inclusos tem como objetivo preservar a saúde bucal, visto que um preceito básico da odontologia atual é a prevenção. A exodontia precoce visa evitar a instalação de doenças de cárie, pericoronarite, reabsorção radicular, má oclusão, cistos e tumores odontogênicos, fratura de mandíbula e dor com etiologia desconhecida, dentre outras possíveis alterações causadas pela presença do dente incluso (Shoshani-Dror et al., 2018; Flor et al., 2021).

Os dentes inclusos não devem ser deixados na cavidade bucal até que causem algum dano ao paciente. A remoção tardia aumenta a morbidade cirúrgica, a perda de tecido ósseo e a possibilidade de lesão de estruturas adjacentes importantes, como o nervo alveolar inferior. Soma-se a estes aspectos a maior incidência de comprometimento sistêmico em pacientes idosos, fato que aumenta os riscos do procedimento cirúrgico (Chou et al., 2017; Ghaeminia et al., 2020).

É importante salientar que nem todos os dentes inclusos estão indicados para exodontia. Em algumas situações, o cirurgião-dentista deve contraindicar a extração destes dentes, a extração está indicada na presença de doenças associadas ao dente e contraindicada na ausência de infecção ou outra condição associada (Peñarrocha-Diago et al., 2021). As contraindicações para a remoção de dentes inclusos envolvem basicamente o estado físico e geral do paciente. Em pacientes debilitados, aguardamos o momento oportuno para a intervenção. Nos processos agudos locais, quando os benefícios potenciais forem maiores que as complicações e riscos potenciais, deve-se realizar o procedimento, caso contrário, o procedimento deve ser adiado (Freitas, 2006; Miloro et al., 2016).

Com intenção de facilitar a comunicação entre os profissionais e evitar os acidentes durante e após as exodontias, foram criadas diversas classificações de dentes inclusos e impactados. As classificações auxiliam o cirurgião dentista no ato cirúrgico, criando assim estratégias para a realização das cirurgias e antecipando o grau de dificuldade de cada uma. As classificações mais populares foram criadas por Winter em 1926 (Winter, 1926), classificando os terceiros molares de acordo com a inclinação do seu longo eixo em relação ao segundo molar, já em 1933 Pell \& Gregory (Pell et al., 1933) classificou os terceiros molares inferiores de duas formas distintas, uma em relação a margem anterior do ramo mandibular e outra relacionada com o plano oclusal. Em 1937 Pell e Gregory descreveram a classificação para os terceiros molares inferiores inclusos, considerando-a em IV etapas, e incorporaram a classificação de Winter na etapa III (Pell et al., 1937; Pell., 1938).

Etapa I - Relação do espaço entre a distal do segundo molar e ramo da mandíbula comparando-o com dimensão da largura da coroa do terceiro molar inferior (Quadro 1).

Quadro 1: Classificação da posição dos terceiros molares segundo Pell e Gregory.

\begin{tabular}{|l|l|}
\hline CLASSE I & $\begin{array}{l}\text { Quando o espaço entre a face distal do segundo molar e o bordo anterior do ramo da mandíbula é maior que a distância } \\
\text { mésio-distal da coroa do terceiro molar incluso. }\end{array}$ \\
\hline CLASSE II & $\begin{array}{l}\text { Quando o espaço entre a face distal do segundo molar e o bordo anterior do ramo da mandíbula é ligeiramente menor do } \\
\text { que a distância mésio-distal da coroa do terceiro molar incluso }\end{array}$ \\
\hline CLASSE III & $\begin{array}{l}\text { Quando o espaço entre a face distal do segundo molar e o bordo anterior do ramo da mandíbula é muito menor do que a } \\
\text { distância mésio-distal da coroa do terceiro molar incluso, ou espaço inexistente, estando grande parte do dente incluso } \\
\text { localizado no ramo da mandíbula. }\end{array}$ \\
\hline
\end{tabular}

Fonte: Adaptado de (Lima et al., 2012; Khojastepour et al., 2019; Bermeo Domínguez et al., 2021) 
Etapa II - Relação de profundidade relativa da coroa do terceiro molar em relação ao plano oclusal do segundo molar adjacente (Quadro 2).

Quadro 2: Classificação da posição dos terceiros molares segundo Pell e Gregory.

\begin{tabular}{|l|l|}
\hline POSIÇÂO A & $\begin{array}{l}\text { Quando a face oclusal do terceiro molar inferior encontra-se na mesma altura ou acima do plano oclusal do segundo } \\
\text { molar. }\end{array}$ \\
\hline POSIÇẤO B & $\begin{array}{l}\text { Quando a face oclusal do terceiro molar inferior está abaixo do plano oclusal, mas acima da linha cervical do segundo } \\
\text { molar. }\end{array}$ \\
\hline POSIÇẤO C & Quando a face oclusal do terceiro molar encontra-se abaixo da linda cervical do segundo molar. \\
\hline
\end{tabular}

Fonte: Adaptado de (Lima et al., 2012; Khojastepour et al., 2019; Bermeo Domínguez et al., 2021).

Etapa III - Relação do terceiro molar inferior com o longo eixo de implantação do segundo molar (Winter, 1926). Pell e Gregory utilizaram a classificação descrita por Winter, levando-se em consideração a angulação dos terceiros molares inferiores inclusos em relação aos segundos molares adjacentes, Winter classificou suas posições em (Quadro 3).

Quadro 3: Classificação da posição dos terceiros molares segundo Winter.

\begin{tabular}{|c|l|}
\hline Mesioangular & Longo eixo do terceiro molar inclinado na direção mesial em relação ao segundo molar. \\
\hline Distoangular & Longo eixo do terceiro molar inclinado na direção distal em relação ao segundo molar. \\
\hline Horizontal & Longo eixo do terceiro molar perpendicular ao longo eixo do segundo molar. \\
\hline Vertical & Longo eixo do terceiro molar paralelo ao longo eixo do segundo molar. \\
\hline Transverso & Dente impactado horizontalmente, mas seu longo eixo na direção vestíbulo-língual ou vestíbulo-palatino. \\
\hline Invertido & O dente está invertido e posicionado de cabeça para baixo. \\
\hline
\end{tabular}

Fonte: Adaptado de (Lima et al., 2012; Khojastepour et al., 2019; Bermeo Domínguez et al., 2021)

Etapa IV - Investigação cuidadosa das possíveis complicações. Retenções, anomalias, dificuldades de acesso e visibilidade.

Segundo Pell e Gregory é recomendado verificas as possíveis retenções, alterações do dente e sua relação com as estruturas anatômicas importantes; alterações patológicas associadas ou próximas ao dente incluso ou ainda a proximidade desses ou de suas raízes com o canal mandibular. Em relação as dificuldades de acesso e visibilidade, o cirurgião dentista de ve estar preparado para atender pacientes com várias alterações anatômicas, além de dificuldade de abertura bucal associada ou não com disfunção da ATM e mialgias. Tudo dependerá da cooperação do paciente, escolha correta da técnica anestésica e cirúrgica. Para alcançar o sucesso na exodontia de dentes inclusos é necessária a realização do planejamento e da intervenção. Com o pré-operatório concluído, diagnosticada a posição e o tipo de inclusão do terceiro molar e analisada as possíveis retenções, anomalias, dificuldades de acesso e visibilidade, o cirurgião dentista poderá analisar a necessidade, oportunidade e assim planejar a intervenção cirúrgica (Neto et al., 2009).

O objetivo deste estudo foi determinar o perfil dos pacientes e dos terceiros molares indicados para exodontia, assim como seu tratamento cirúrgico realizado na faculdade de Odontologia de Araçatuba - UNESP, avaliando a prevalência das posições de terceiros molares em relação às classificações de Winter e Pell \& Gregory.

\section{Metodologia}

O estudo foi baseado na aplicação de fichas em forma de questionário aplicadas pelos alunos voluntários do projeto de extensão “Clínica de atualização para extração dos dentes não erupcionados e impactados" realizado na Faculdade de Odontologia de Araçatuba pelo Departamento de Cirurgia e Clínica integrada nos anos de 2014 a 2016 . A ficha aplicada foi 
composta pela identificação do paciente e anamnese, identificação do dente incluso, técnica da exodontia, acidentes e complicações. A ficha foi preenchida para cada dente extraído e o procedimento cirúrgico foi realizado por alunos da graduação (Figura 1).

Figura 1: Ficha utilizada para o estudo.

Projeto extensão “Clínica de atualização para extração dos dentes não erupcionados e impactados”.

\section{IDENTIFICAÇÃO E ANAMNESE.}

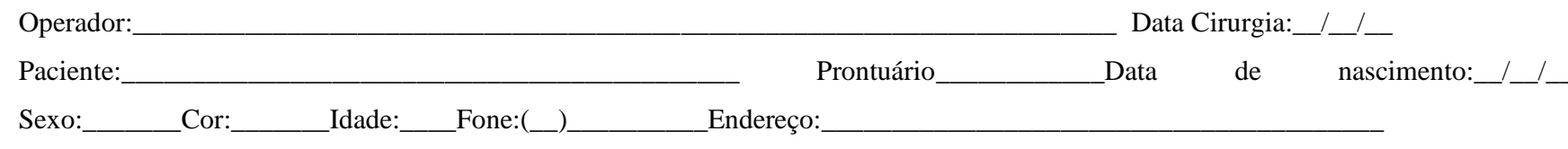

Cidade: Problemas Sistêmicos: ( ) Nega ( ) Sim:

\section{IDENTIFICAÇÃO DO DENTE INCLUSO.}

Terceiro Molar (18) (28) (38) (48).

Canino (13) (23) (33) (43).

Pré-Molar:

Supranumerário (localização): Outro:

Erupcionado: ; Incluso: ; Semi-incluso:

Winter 1926: ( ) Vertical, ( ) Mesio Angular, ( ) Horizontal, ( ) Disto Angular, ( ) Invertido ( ) Transverso

Pell \& Gregory: Posição: ( ) A, ( ) B, ( ) C. Classe: ( ) 1, ( ) 2, ( ) 3.

Transtornos: ( ) Nega ( ) Sim

Transtornos mecânicos: ( ) Mal oclusão, ( ) Reabsorção radicular, ( ) Fratura Óssea, ( ) Mordida Cruzada Posterior, ( ) Desalinhamento Dental, ( ) Pericementite, ( ) Diastema Interdental, ( ) Deslocamento de prótese, ( ) Trauma da mucosa Jugal ou da gengiva.

Transtornos infecciosos: ( ) Pericoronarite, ( ) Abscesso, ( ) Cárie, ( )Sinusite, ( ) Trismo.

Transtornos nervosos: ( ) Dor, ( ) Disfunção de ATM, ( ) Alopecia areata.

Transtornos tumorais: ( ) Neoplasias Benignas, ( ) Cistos, ( ) Neoplasias Malignas

TÉCNICA DA EXODONTIA: ( ) Simples ( ) Retalho ( ) Osteotomia ( ) Ostectomia

( ) Odontosecção_

ACIDENTES E COMPLICAÇÕES: ( ) Desgarramento da mucosa, ( ) Fratura de túber, ( ) Lesão de dentes vizinhos,

( ) Luxação da mandíbula, ( ) Comunicação Buco-Sinusal, ( ) Penetração de raiz no seio maxilar, ( ) Penetração de dente na via respiratória, ( ) Lesão de tecidos moles, ( ) Parestesia do n. Lingual, ( ) Fratura radicular, ( ) Alergia, ( ) Edema( ) Hematomas, ( ) Hemorragia, ( ) Alveolite, ( ) Penetração de dente na via digestiva, ( ) Infecção ( ) Parestesia, ( ) Fratura de mandíbula, ( ) Parestesia do n. Alveolar Inferior.

Fonte: Autores.

Para a análise da classificação dos terceiros molares, os alunos da graduação, avaliaram as radiografias panorâmicas solicitadas para cada paciente e aplicaram a classificação de Pell e Gregory para os terceiros molares inferiores e a classificação de Winter para os terceiros molares superiores e inferiores, preenchendo as fichas utilizadas para o estudo. 
A análise das fichas foi realizada por apenas um examinador e os dados foram catalogados, organizados e apresentados com auxílio do programa Excel (Microsoft $®$ ), nos computadores da Disciplina de Cirurgia e Traumatologia Bucomaxilofacial da Faculdade de Odontologia de Araçatuba - UNESP.

\section{Resultados}

Os resultados encontrados no presente estudo demonstram o perfil dos pacientes atendidos na Faculdade de Odontologia de Araçatuba e dos procedimentos realizados pelos alunos da graduação. Como podemos observar no estudo, o número de pacientes atendidos ao longo dos três anos foi de 134, sendo eles 77 do sexo feminino e 57 do sexo masculino. $\mathrm{O}$ que reflete uma porcentagem de $57 \%$ de pacientes mulheres atendidas, sendo a maioria dos atendimentos, e $43 \%$ de pacientes homens (Gráfico 1).

Gráfico 1: Gênero dos pacientes.

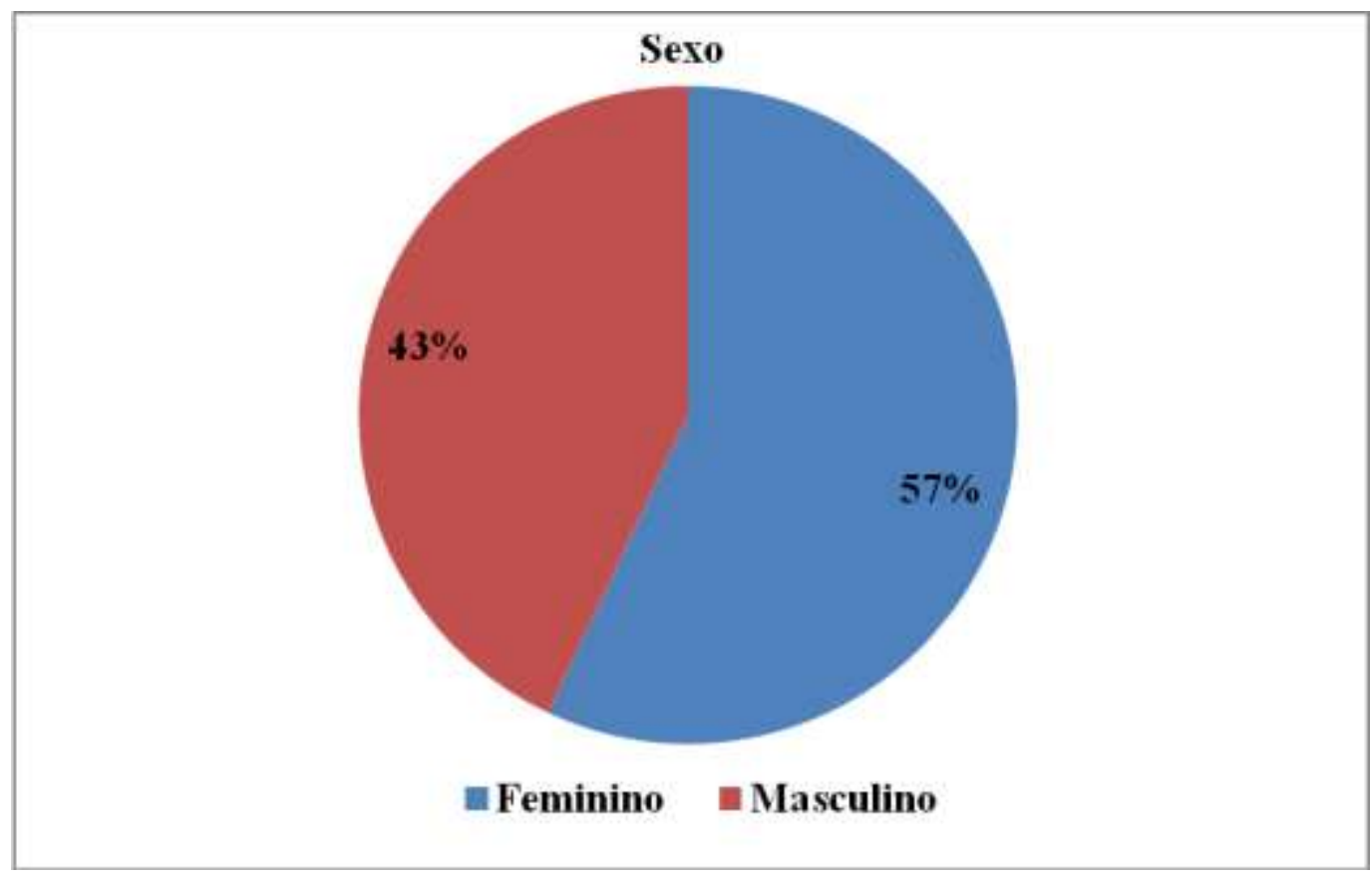

Fonte: Autores.

Quando observamos a faixa etária dos pacientes a maior procura por atendimento é feita por pessoas entre 20 e 29 anos sendo composta por $60 \%$ dos pacientes ( 80 pacientes), seguida por pacientes com idades entre 30 e 39 anos com $20 \%$ (27 pacientes), pacientes com menos de 20 anos representaram 13\% (17 pacientes). Pacientes com a faixa etária entre 40 a 44 anos ( 3 pacientes) e 45 a 49 anos ( 3 pacientes) obtiveram porcentagem de $2 \%$ seguida pela faixa etária de acima de 50 anos com 4 pacientes compondo 3\% (Quadro 4 e Gráfico 2). 
Research, Society and Development, v. 10, n. 11, e358101119770, 2021

(CC BY 4.0) | ISSN 2525-3409 | DOI: http://dx.doi.org/10.33448/rsd-v10i11.19770

Quadro 4: Idade e quantidade de pacientes atendidos.

\begin{tabular}{|l|l|}
\hline Idade & Quantidade \\
\hline 20 a 29 anos & 80 pacientes \\
\hline 30 a 39 anos & 27 pacientes \\
\hline Menos de 20 anos & 17 pacientes \\
\hline Mais de 50 anos & 4 pacientes \\
\hline 40 a 44 anos & 3 pacientes \\
\hline 45 a 49 anos & 3 pacientes \\
\hline
\end{tabular}

Fonte: Autores.

Gráfico 2: Idade dos pacientes atendidos em porcentagem.

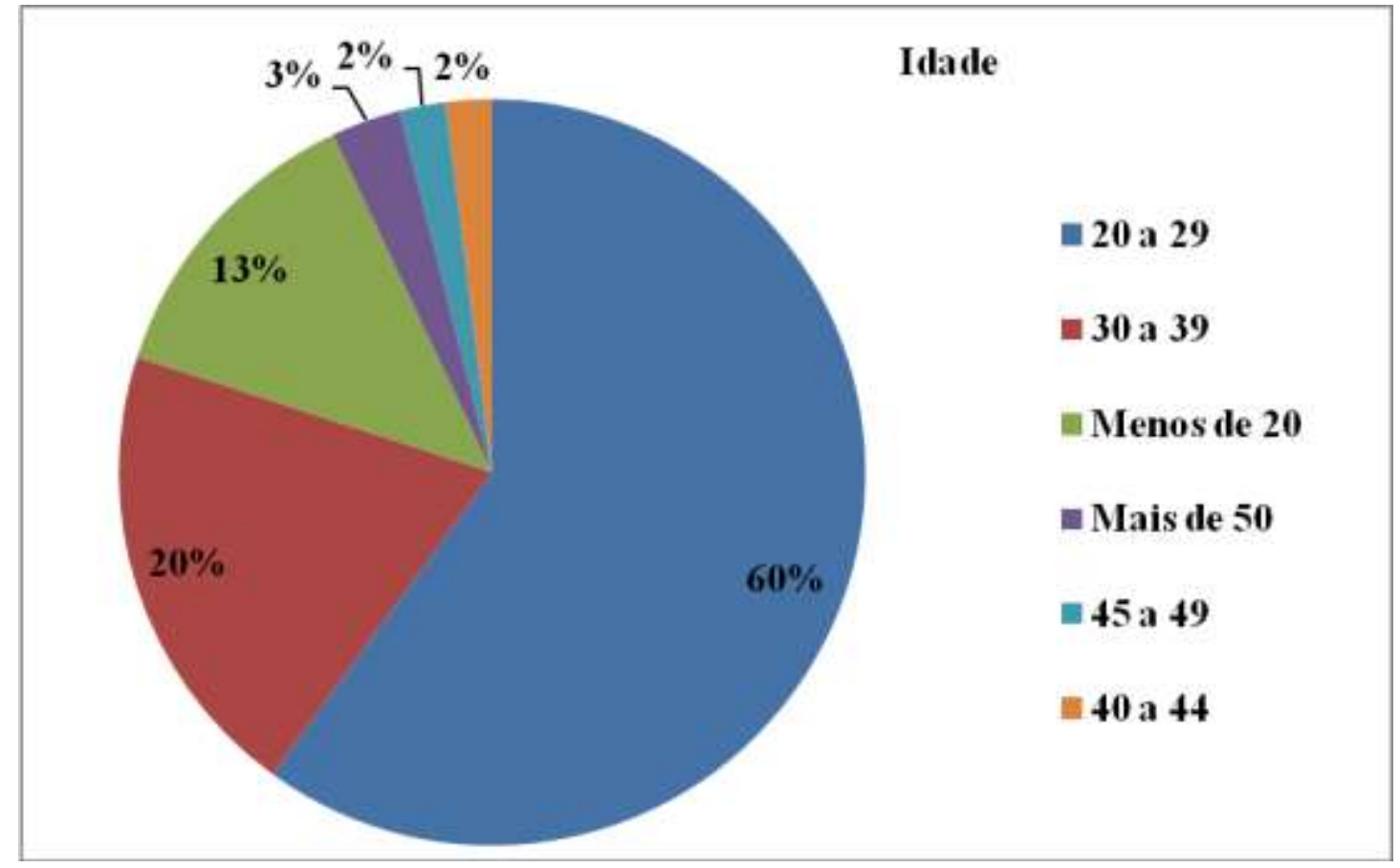

Fonte: Autores.

Dos 134 pacientes atendidos, apenas 13 pacientes relataram alterações sistêmicas. Dos pacientes com alterações sistêmicas a rinite foi a alteração com maior número de casos (Quadro 5). 
Quadro 5: Alterações sistêmicas dos pacientes.

\begin{tabular}{|l|l|}
\hline Rinite & 3 casos \\
\hline Anemia & 1 caso \\
\hline Gastrite & 1 caso \\
\hline Púrpura trombocitopênica & 1 caso \\
\hline Asma & 1 caso \\
\hline Febre reumática & 1 caso \\
\hline Lúpus eritematoso & 1 caso \\
\hline Deficiência vitamínica & 1 caso \\
\hline Alteração no sono & 1 caso \\
\hline Diabetes & 1 caso \\
\hline HIV positivo & 1 caso \\
\hline
\end{tabular}

Fonte: Autores.

No estudo foram realizadas 275 exodontias de terceiros molares, o dente que obteve maior número de exodontias foi o dente 48 com 82 procedimentos cirúrgicos (30\% de todos os procedimentos realizados), seguido do dente 18 com 73 casos ( $26 \%$ dos procedimentos realizados) e dos dentes 38 com 65 casos ( $24 \%$ dos procedimentos realizados) e dentes 28 com 55 casos (20\% dos procedimentos realizados) (Quadro 6 e Gráfico 3).

Quadro 6: Exodontias realizadas.

\begin{tabular}{|l|l|}
\hline Dente 48 & 82 casos \\
\hline Dente 18 & 73 casos \\
\hline Dente 38 & 65 casos \\
\hline Dente 28 & 55 casos \\
\hline
\end{tabular}

Fonte: Autores. 
Gráfico 3: Porcentagem de exodontias realizadas.

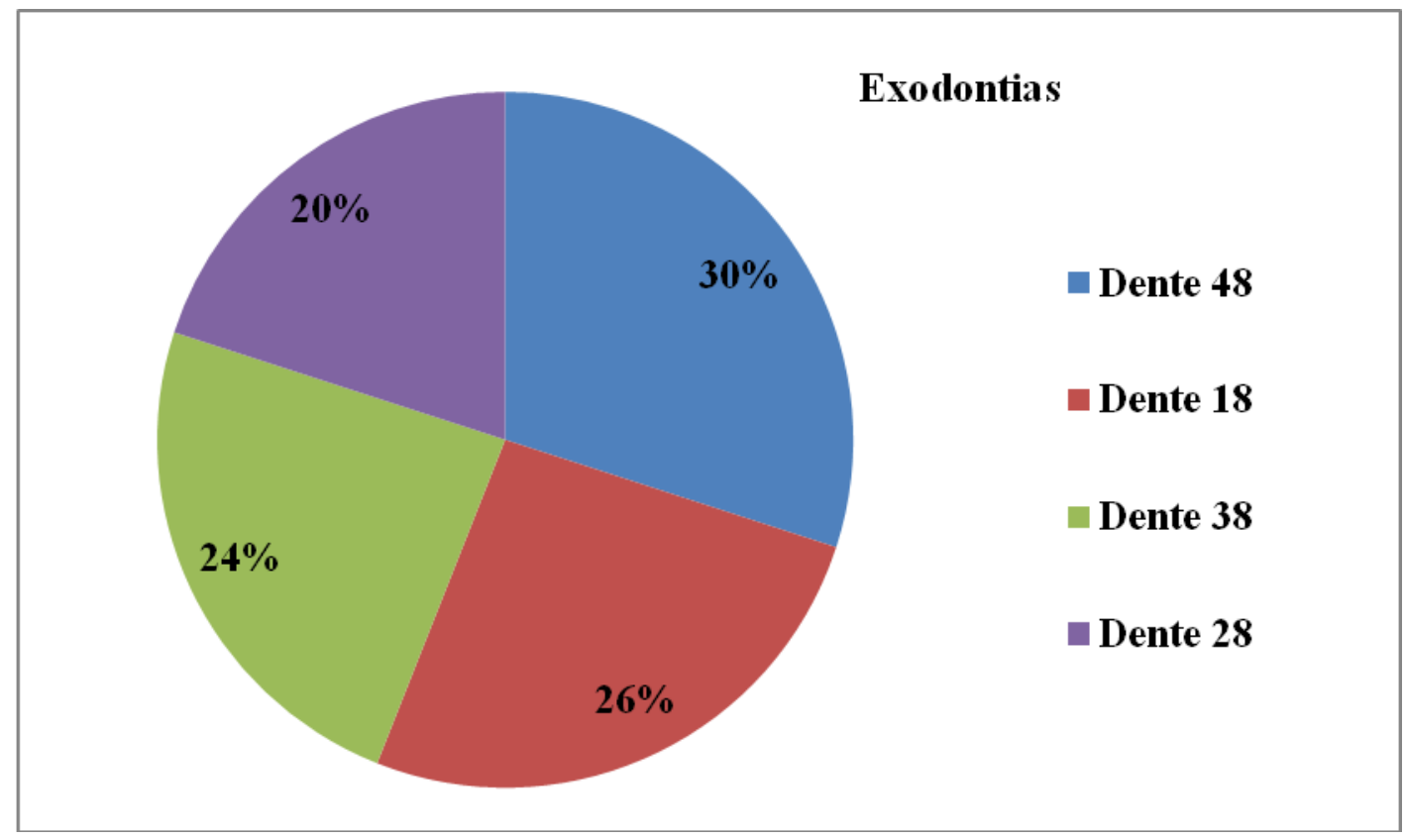

Fonte: Autores.

Em relação a técnica cirúrgica utilizada nas exodontias os dados mostram que foram realizados 81 casos com a técnica simples de exodontia, 182 casos com retalho gengival, 152 casos de osteotomia, 29 casos de ostectomia e 124 casos onde foram realizadas odontosecções. Para cada dente realizou-se um conjunto de técnicas cirúrgicas para alcançar o objetivo da exodontia (Gráfico 4).

Gráfico 4: Técnica cirúrgica utilizada nas exodontias.

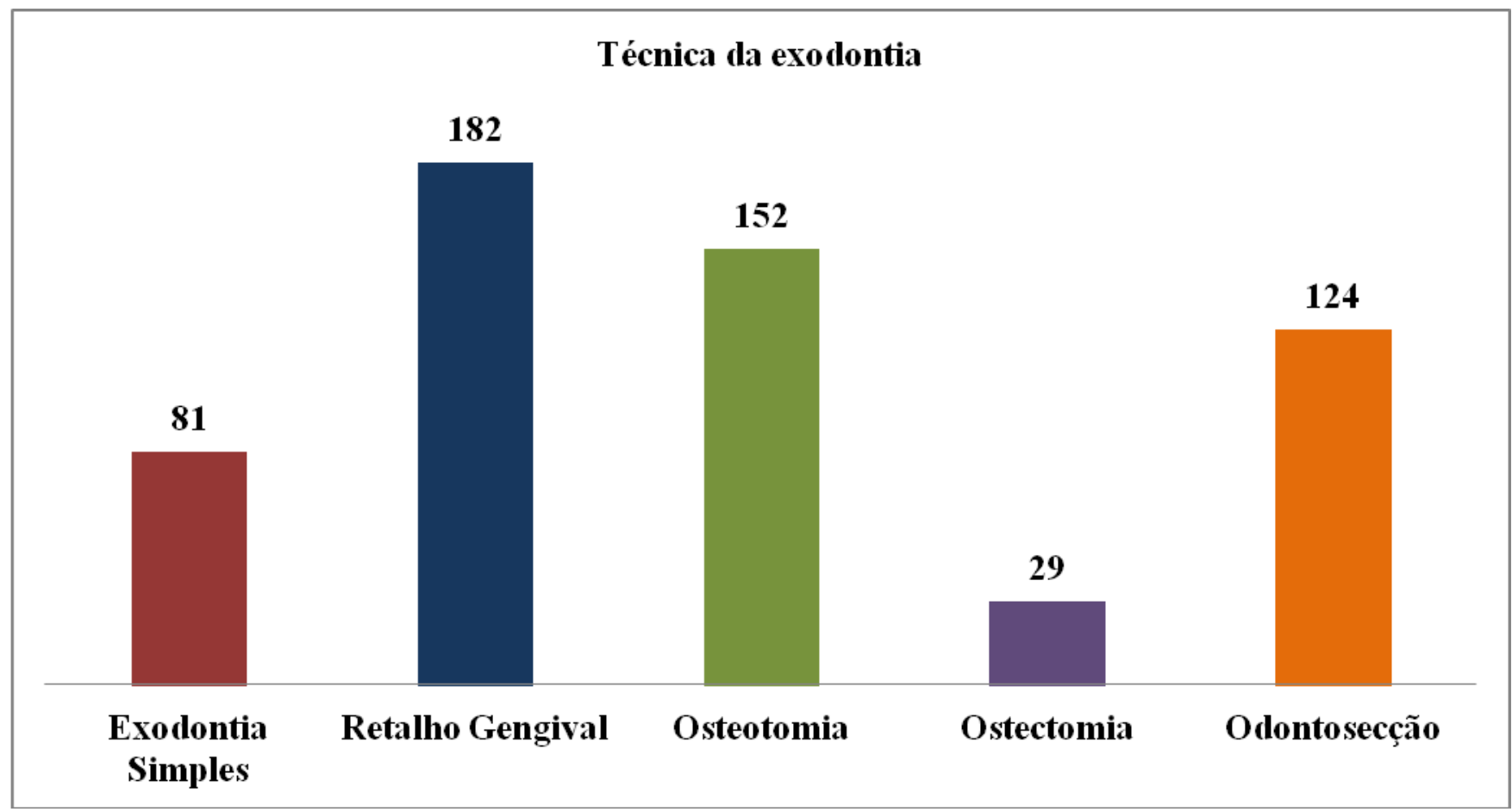

Fonte: Autores. 
Segundo a Classificação de Pell e Gregory aplicada para os 147 terceiros molares inferiores, a prevalência no estudo relacionado a margem anterior do ramo mandibular, foi de terceiros molares na Posição A com 64 casos (43,5\%), seguido pela Posição B com 62 casos (42,2\%) e Posição C com 21 (14,3\%). Em relação a classificação levando-se em consideração o plano oclusal, a prevalência foi de terceiros molares na Classe II com 71 casos (48,3\%), seguido pela Classe I com 65 casos $(44,2 \%)$ e pela Classe III com 11 casos (7,5\%) (Quadro 7 e Gráfico 5).

Quadro 7: Dentes classificados segundo a classificação de Pell e Gregory

\begin{tabular}{|l|l|}
\hline Posição A & 64 casos \\
\hline Posição B & 62 casos \\
\hline Posição C & 21 casos \\
\hline
\end{tabular}

\begin{tabular}{|c|l|}
\hline Classe II & 71 casos \\
\hline Classe I & 65 casos \\
\hline Classe III & 11 casos \\
\hline
\end{tabular}

Fonte: Autores.

Gráfico 5: Porcentagem de dentes classificados segundo a classificação de Pell e Gregory.

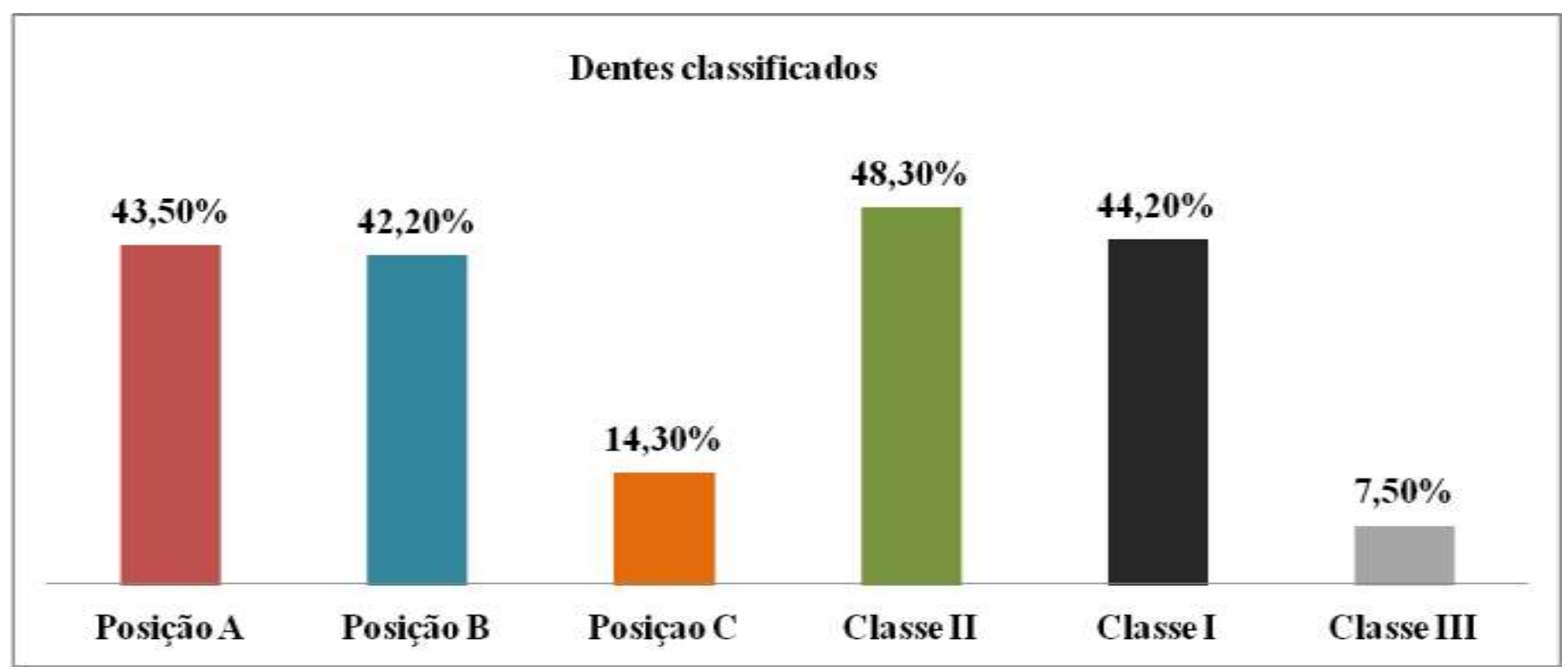

Fonte: Autores.

Segundo a Classificação de Winter de 1926, aplicada para os 275 terceiros molares superiores e inferiores, a prevalência no estudo foi de dentes na posição vertical com 160 casos (58\%), seguido pela posição mésioangular com 74 casos (27\%), horizontal com 33 casos (12\%), distoangular com 8 casos (3\%) e nenhuma citação sobre terceiro molar invertido ou transverso (Quadro 8 e Gráfico 6). 
Research, Society and Development, v. 10, n. 11, e358101119770, 2021

(CC BY 4.0) | ISSN 2525-3409 | DOI: http://dx.doi.org/10.33448/rsd-v10i11.19770

Quadro 8: Classificação de Winter 1926.

\begin{tabular}{|l|l|}
\hline Vertical & 160 casos \\
\hline Mésioangular & 74 casos \\
\hline Horizontal & 33 casos \\
\hline Distoangular & 8 casos \\
\hline Invertido & 0 casos \\
\hline Transverso & 0 casos \\
\hline
\end{tabular}

Fonte: Autores.

Gráfico 6: Porcentagem de dentes classificados segundo a classificação de Winter 1926.

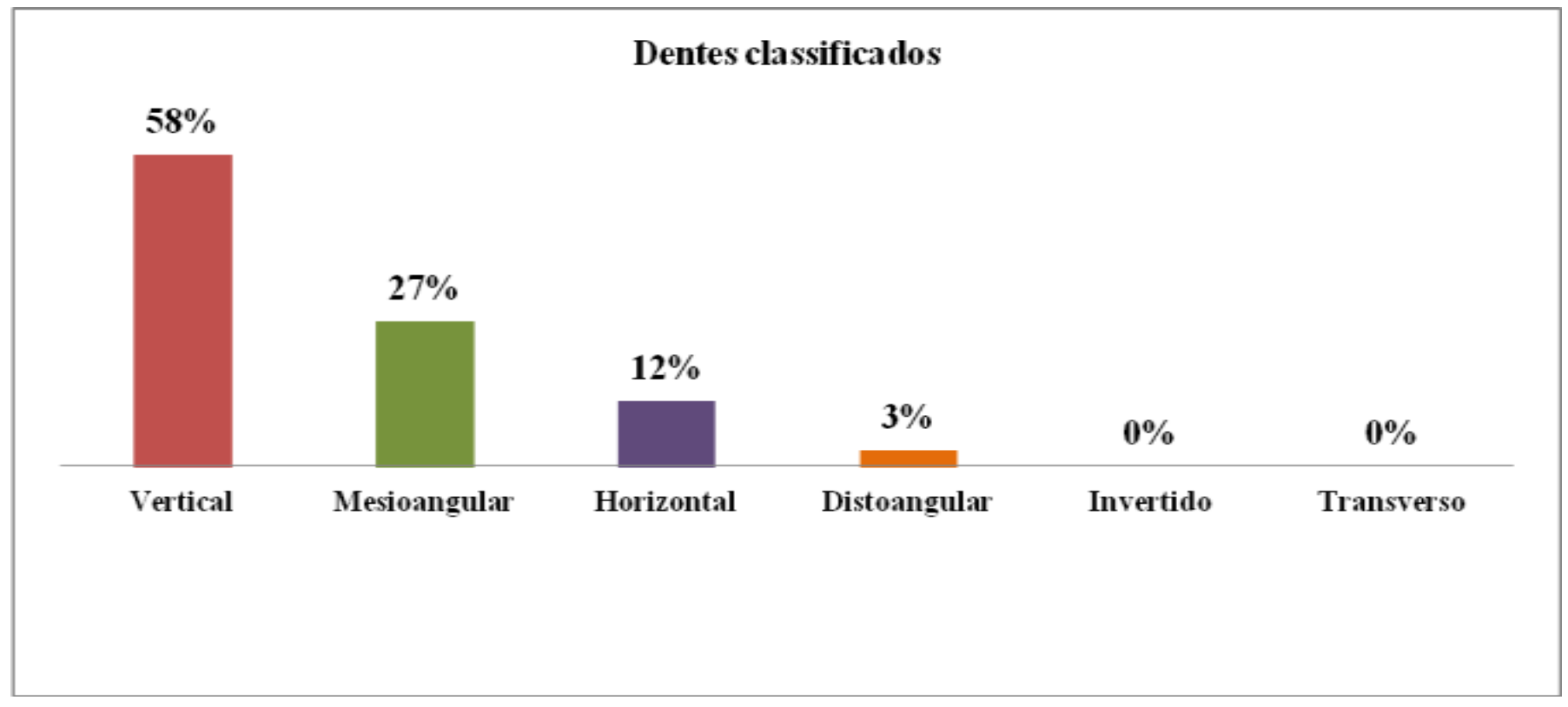

Fonte: Autores.

No presente estudo foram observados alguns transtornos quando observamos o tratamento e procedimento dos terceiros molares (Quadro 9). 
Quadro 9: Transtornos relatados.

\begin{tabular}{|c|c|}
\hline \multicolumn{2}{|c|}{ Transtornos Mecânicos } \\
\hline Transtornos & Número de casos \\
\hline Má oclusão & 31 casos \\
\hline Trauma de mucosa jugal ou gengival & 8 casos \\
\hline Pericementite & 7 casos \\
\hline Reabsorção radicular & 6 casos \\
\hline Fratura óssea & 1 caso \\
\hline Diastema interdental & 1 caso \\
\hline Desalinhamento dental & 1 caso \\
\hline \multicolumn{2}{|c|}{ Transtornos Infecciosos } \\
\hline Transtornos & Número de casos \\
\hline Pericoronarite & 47 casos \\
\hline Cárie & 9 casos \\
\hline Trismo & 2 casos \\
\hline \multicolumn{2}{|c|}{ Transtornos Nervosos } \\
\hline Transtornos & Número de casos \\
\hline Dor & 77 casos \\
\hline Disfunção da ATM & 6 casos \\
\hline \multicolumn{2}{|c|}{ Transtornos Tumorais } \\
\hline
\end{tabular}

Fonte: Autores.

No estudo foi possível observar alguns acidentes trans-cirúrgicos e complicações pós-cirúrgicas, sendo o edema, parestesia do nervo alverolar inferior e alveolite os mais frequentes (Quadro 10).

Quadro 10: Acidentes e complicações pós-cirúrgicas.

\begin{tabular}{|l|l|}
\hline Edema & 32 casos \\
\hline Comunicação buco-sinusal & 4 casos \\
\hline Hemorragia & 3 casos \\
\hline Fratura de túber & 3 casos \\
\hline Parestesia Nervo Alveolar inferior & 6 casos \\
\hline Parestesia Nervo lingual & 1 caso \\
\hline Desgarramento de mucosa & 3 casos \\
\hline Fratura radicular & 3 casos \\
\hline Alveolite & 6 casos \\
\hline Hematomas & 1 caso \\
\hline Lesão de tecidos moles & 3 casos \\
\hline Parestesia & 2 casos \\
\hline
\end{tabular}

Fonte: Autores. 


\section{Discussão}

A etiologia da inclusão dental dos terceiros molares é multifatorial, podendo estar associada a hereditariedade, alterações patológicas, alterações sistêmicas e traumatismos, ressaltando-se a falta de espaço para sua acomodação, como um dos fatores mais importantes (Cerqueira et al., 2007). Os indivíduos pertencentes a civilização moderna, apresentam uma diminuição do arcabouço ósseo, maxila e mandíbula com consequente diminuição do espaço disponível para a acomodação dos terceiros molares. Esta tendência evolutiva pode estar ligada a variação racial, a natureza da dieta, o grau de uso do aparelho mastigatório e a herança genética, afetando o desenvolvimento ósseo dos maxilares (Hattab et al., 1995; Sandhu et al., 2005).

Comprovando esses fatos, a agenesia dos quartos e terceiros molares têm aumentado ultimamente (Marzola et al., 1968). A agenesia de terceiros molares ocorre na frequência de $14,42 \%+/-1,72$, sendo de $13,42 \%$ no sexo masculino, e de $15,21 \%$ no sexo feminino, sem diferenças significativas. Incide em 51,42\% +/- 4,22 nos terceiros molares superiores e em 48,57\% +/- 4,22 nos inferiores, sem diferenças significativas (Marzola et al., 1968).

No estudo realizado foram atendidos 134 pacientes sendo 77 do sexo feminino (57\%) e 57 do sexo masculino (43\%), sendo o sexo feminino com maior incidência e compatível com o estudo realizado por Garcia (Garcia et al., 2010) e Marzola (Marzola et al., 1968), onde afirmam a maior prevalência do sexo feminino em casos de exodontia de terceiros molares. Sugere-se que o maior percentual de pacientes do gênero feminino nesta pesquisa seja devido a maior preocupação desde grupo com a saúde bucal, motivando uma maior procura aos serviços de saúde.

Quanto à prevalência da idade dos pacientes, foi observado no estudo maior incidência da faixa etária de 20 a 29 anos com 80 pacientes, $60 \%$ dos atendimentos seguida da faixa etária de 30 a 39 anos com 27 pacientes, 20\%. Resultado que é compatível com os estudos de Marinho (Marinho et al., 2005), que aponta a prevalência de terceiros molares na faixa de idade acima encontradas.

Em relação aos terceiros molares inclusos, autores como Dias-Ribeiro (Dias-Ribeiro et al., 2008) e Marzola (Marzola et al., 1968), citam que há uma maior prevalência de retenção dos terceiros molares inferiores sobre os superiores. No estudo realizado, o resultado entre os 275 dentes extraídos é semelhante as citações dos autores acima, os dentes inferiores 38 e 48 somaram $54 \%$ (147 dentes) seguidos pelos superiores 18 e 28 com $46 \%$ (128 dentes).

Entre os 275 terceiros molares superiores e inferiores analisados e classificados segundo Winter (1926), sua maior prevalência foi a posição vertical com 160 casos (58\%), seguido da posição mésio-angular com 74 casos (27\%), horizontal com 33 casos (12\%), disto-angular com 8 casos (3\%) e nenhum caso invertido e ou transverso realizado. O estudo é compatível com autores como Farias (Farias et al., 2003) e Cerqueira (Cerqueira et al., 2007), que citam a posição vertical como a mais frequentemente encontrada.

Em relação a classificação de Pell e Gregory (1933), aplicada para os 147 terceiros molares inferiores analisados, em relação ao plano oclusal, a posição A apresentou a maior prevalência, assim como também encontrada nos estudos de Costa (Costa et al., 2010) e Santos (Santos et al., 2009). Já em relação a inclusão no ramo mandibular, a Classe II foi a mais prevalente, assim como nos estudos de Trento (Trento et al., 2009) e Xavier (Xavier et al., 2010).

Uma vez indicada a exodontia do terceiro molar é fundamental a realização de um planejamento cirúrgico baseado nos exames clínico e radiográfico com o intuito de prevenir possíveis complicações no trans e pós-operatório. No presente estudo foram observados transtornos mecânicos, infecciosos, nervosos, acidentes trans-cirúrgicos e complicações póscirúrgicas. Entre os transtornos observados, os que tiveram maior prevalência foram a dor (77 casos), pericoronarite (47 casos), má oclusão (31 casos), e cárie (9 casos). Entre os acidentes e complicações os mais comuns foram o edema (32 casos), alveolite (6 casos) e parestesia no nervo alveolar inferior (6 casos). 


\section{Conclusão}

A maioria dos pacientes submetidos a exodontia dos terceiros molares na Faculdade de Odontologia de Araçatuba UNESP são jovens do sexo feminino. Prevalecem os procedimentos de exodontia de terceiros molares inferiores em posição A, classe II e vertical, segundo a classificação de Pell e Gregory (1933) e Winter (1926). As complicações operatórias mais frequentes foram o edema, alveolite e parestesia do nervo alveolar inferior.

\section{Referências}

Bermeo Domínguez, J. B., Morales González, P. M., \& Bravo Calderón, M. E. (2021). Analysis of third molars and their adjacent anatomic structures by means of CBCT: meta-analysis. Research, Society and Development. 10 (11), e226101119723.

Cerqueira, P. R. F., Farias, D. L. B., Silva Filho, J. P., \& Oliveira, T. Q. F. (2007). Análise da topografia axial dos terceiros molares inclusos através da radiografia panorâmica dos maxilares em relação à classificação de Winter. Rev Odonto Ciênc. 22 (55), 16-22.

Chou, Y. H., Ho, P. S., Ho, K. Y., Wang, W. C., \& Hu, K. F. (2017). Association between the eruption of the third molar and caries and periodontitis distal to the second molars in elderly patients. Kaohsiung J Med Sci. 33 (5), 246-251.

Costa, M. P., Oliveira, A. F., Costa, J. F., Silva, R. A., Lopes, F. F., \& Silva, A. B. (2010). Incidência das Posições Anatômicas e Agenesia dos Terceiros Molares em Estudantes de São Luís, Maranhão. Pesq. Bras. Odontoped. Clin. Integr. 10 (3), 399-403.

Dias-Ribeiro, E., Lima-Júnior, J. L., Barbosa, J. L., Haagsma, I. B., Lucena, L. B. S., \& Marzola, C. (2008). Evaluation of the positions of retained third molars in relation of Winter's classification. Rev Odontol UNESP. 37 (3), 203-209.

Farias, J. G., Santos, F. A. P., Campos, P. S. F., Sarmento, V. A., Barreto, S., \& Rios, V. (2003). Prevalência de dentes inclusos em pacientes atendidos na disciplina de cirurgia do curso de Odontologia da Universidade Estadual de Feira de Santana. Pesq Bras Odontoped Clin Integr. 3 (2), 15-19.

Flor, L. C. S., Trinta, L. B., Gomes, A. V. S. F., Figueiredo, R. B., Sousa, A. C. A., Silva, L. C. N., Gomes, F. S., Freire, M. D. P., \& Agostinho, C. N. L. F. (2021). Factors associated with accidents and complications on third molar extraction: a literature review. Research, Society and Development. 10 (10), e281101018932.

Freitas, R. Tratado de cirurgia bucomaxilo facial. (2006). Ed. Santos.

Garcia, R. R., Paza, A. O., Moreira, R. W., Moraes, M., \& Passeri, L. A. (2010). Avaliação radiográfica da posição de terceiros molars inferiores segundo as classificações de Pell \& Gregory e Winter. Revista Da Faculdade De Odontologia - UPF. 5 (2), 31-36.

Ghaeminia, H., Nienhuijs, M. E., Toedtling, V., Perry, J., Tummers, M., Hoppenreijs, T. J., Van der Sanden, W. J., \& Mettes, T. G. (2020). Surgical removal versus retention for the management of asymptomatic disease-free impacted wisdom teeth. Cochrane Database Syst Rev. 5 (5), CD003879.

Hattab, F. N., Rawashdeh, M. A., \& Fahmy, M. S. (1995). Impaction status of third molars in Jordanian students. Oral Surg Oral Med Oral Pathol Oral Radiol Endod. 79 (1), 24-29.

Khojastepour, L., Khaghaninejad, M. S., Hasanshahi, R., Forghani, M., \& Ahrari, Farzaneh.(2019). Does the Winter or Pell and Gregory Classification System Indicate the Apical Position of Impacted Mandibular Third Molars? Journal of Oral and Maxillofacial Surgery.77 (2222), e1-2222.e9.

Lima, C. J., Silva, L. C. F., Melo, M. R. S., Santos, J. A. S. S., \& Santos, T. S.(2012). Evaluation of the agreement by examiners according to classifications of third molars. Med Oral Patol Oral Cir Bucal. 17 (2), e281-6.

Marinho, S. A., Verli, F. D., Amenabar, J. M., \& Brucker, M. R. (2005). Avaliação da posição dos terceiros molares inferiores retidos em radiografias panorâmicas. ROBRAC. 14 (37), 65-68.

Marzola, C., Madeira, M. C., \& Castro, A. L.(1968). Ocorrência de retenção e agenesia dental em 1760 indivíduos. Arq Cent Est Fac UFMG. 5 (1), $33-46$.

Medeiros, P. J., Miranda, M. S., Ribeiro, D. P. B., Louro, R. S., \& Moreira, L. M. (2003).Cirurgia dos dentes inclusos: extração e aproveitamento. Santos.

Miloro, M., Ghali, G. E., Larsen, P. E., \& Waite, P. D. (2016). Princípios de cirurgia bucomaxilofacial de Peterson. (3a ed.), Santos Editora.

Neto, M. S., Filho, O. M., Júnior, I. R. G., Aranega, A., \& Fattah, C. M. R. S. (2009). Tratamento cirúrgico dos dentes não erupcionados. Araçatuba: Faculdade de Odontologia de Araçatuba. 53-56.

Pell, G. J. (1938). Classification and technic for the removal of impacted mandibular third molars. J.A.D.Ass.Dent.Cos. 25 (10), $1594-1597$.

Pell, G. J., \& Gregory, B. T. (1933). Impacted mandibular third molars classification and modified technique for removal. Dental Dig. 39, 330-8.

Pell, G. J., \& Gregory, G. T. (1937). A classification of impacted mandibular third molars. J.dent. Educat. 1 (4), 157-60.

Peñarrocha-Diago, M., Camps-Font, O., Sánchez-Torres, A., Figueiredo, R., Sánchez-Garcés, M. A., \& Gay-Escoda, C. (2021). Indications of the extraction of symptomatic impacted third molars. A systematic review. J Clin Exp Dent.13 (3), e278-e286.

Sandhu, S., \& Kaur, T.(2005). Radiographic evaluation of the status of third molars in the Asian-Indian students. J Oral Maxillofac Surg. 63 (5), $640-645$. 
Research, Society and Development, v. 10, n. 11, e358101119770, 2021

(CC BY 4.0) | ISSN 2525-3409 | DOI: http://dx.doi.org/10.33448/rsd-v10i11.19770

Santos, D. R., \& Quesada, G. A. T. (2009). Prevalência de terceiros molares e suas respectivas posições segundo as classificações de Winter e de Pell e Gregory. Rev. Cir. Traumatol. Buco-Maxilo-fac. 9 (1), 83-92.

Shoshani-Dror, D., Shilo, D., Ginini, J. G., Emodi, O., \& Rachmiel, A. (2018). Controversy regarding the need for prophylactic removal of impacted third molars: An overview. Quintessence Int. 49 (8), 653-662.

Trento, C. L., Zini, M. M., Moreschi, E., Zamponi, M., Gottardo, D. V., \& Cariani, J. P. (2009). Localização e classificação de terceiros molares: análise radiográfica. Interbio. 3 (2), 18-26.

Winter, G. B. (1926). Impacted mandibular third molar. St. Louis: American Medical Book.

Xavier, C. R. G., Dias-Ribeiro, E., Ferreira-Rocha, J., Duarte, B. G., Ferreira-Júnior, O., Sant'Ana, E., \& Gonçales, E. S. (2010). Evaluation of the positions of impacted third molars according to the Winter and Pell \& Gregory classifications in panoramic radiography. Rev.cir.traumatol.buco-maxilo-fac. 10 (2), 83-90. 